\title{
Contributions of the escape depth to the photoelectron intensity of a well-defined initial state
}

\author{
M. Morgenstern, ${ }^{1}$ T. Strasser, ${ }^{2}$ R. Adelung, ${ }^{3}$ M. Getzlaff, ${ }^{4}$ L. Kipp, ${ }^{3}$ M. Skibowski, ${ }^{3}$ W. Schattke, ${ }^{2,5}$ and R. Wiesendanger ${ }^{1}$ \\ ${ }^{1}$ Institute of Applied Physics, Hamburg University, Jungiusstraße 11, D-20355 Hamburg, Germany \\ ${ }^{2}$ Institut für Theoretische Physik und Astrophysik, Christian-Albrechts-Universität, Leibnizstraße 15, D-24098 Kiel, Germany \\ ${ }^{3}$ Institute for Experimental and Applied Physics, Christian-Albrechts-University, Leibnizstraße 19, D-24098 Kiel, Germany \\ ${ }^{4}$ Institute of Applied Physics, University of Düsseldorf, Universitätsstr. 1, D-40225 Düsseldorf, Germany \\ ${ }^{5}$ Donostia International Physics Center (DIPC), Manuel de Lardizabal 4, E-20018 San Sebastian, Spain
}

(Received 19 April 2004; published 25 August 2004)

\begin{abstract}
Using the adsorbate-induced two-dimensional electron system on $\operatorname{InAs}(110)$ as an initial state, which provides rather small energy and $k$-space distribution, we measure the photoelectron intensity as a function of photoelectron energy. It oscillates twice between $3 \mathrm{eV}$ and $23 \mathrm{eV}$ having maxima at $5.5 \mathrm{eV}$ and $19 \mathrm{eV}$. Comparison with calculations performed within the one-step model shows that the maximum at low energy is due to an overlap of a maximum in the photoelectron escape depth and a maximum of the matrix elements corresponding to the atomic wave functions. In contrast, the maximum at high energy is caused by final states crossing the $\Gamma$ point exactly at this energy. The comparison confirms the theoretical prediction that the escape depth at low excitation energies can be significantly modified by band-structure effects.
\end{abstract}

DOI: 10.1103/PhysRevB.70.081305

PACS number(s): 79.60.Jv, 73.21.Fg

Angle-resolved photoelectron spectroscopy (ARUPS) at energies between 10 and $100 \mathrm{eV}$ is commonly used to map the band structure of occupied electronic states. At these energies ARUPS is extremely surface sensitive. At higher energies above $1 \mathrm{keV}$, the information depth is increased and ARUPS becomes more bulk sensitive. ${ }^{1}$ At energies below $10 \mathrm{eV}$, one generally assumes that the escape depth $\lambda(E)$ increases monotonically, following a material independent universal curve. ${ }^{2}$ However, deviations from the universal curve are found for several materials. ${ }^{3}$ Partly, a local maximum in $\lambda(E)$ exists at low energy, e.g., for $\mathrm{Rb}, \mathrm{Cs},{ }^{4}$ or $\mathrm{SiO}_{2}{ }^{5}$ The reduction of $\lambda(E)$ below the maximum is explained by additional scattering from $d$ electrons or phonons. Recent calculations found such a maximum also for III-V materials ${ }^{6}$ and explained it by an increase of $\lambda$ at critical points of the band structure, indicating a relevant interaction between decaying final states and bulk bands.

In order to verify the predicted maximum experimentally, one has to consider that the photoelectron intensity depends also on matrix elements and the relevant density of final states. ${ }^{7}$ To disentangle the different contributions, it is useful to start with a well-defined initial state. Therefore, we use the Fe-induced two-dimensional electron system (2DES) close to the surface of $\operatorname{InAs}(110) .{ }^{8,9}$ We find excellent agreement between the measured and the calculated energy dependence of photoelectron intensity. From comparison with the onestep photoemission calculations, we indeed deduce an oscillation of $\lambda(E)$ at low kinetic energies.

The advantages of our initial state are the following:

(1) The 2DES is energetically separated from all other states of the sample, since it exists in the bulk band gap, i.e., above all occupied bulk states. ${ }^{8,9}$ This eliminates influences of energetic overlap with photoelectrons from other bands.

(2) The 2DES belongs to an electronically simple $s$-like and nearly parabolic band, ${ }^{10}$ which reduces the complexity of matrix element effects.

(3) The 2DES electrons are restricted to $\vec{k} \simeq \overrightarrow{0}$, reducing the number of relevant final states significantly. Parallel to the surface $(x, y)$ only the inner $0.2 \%$ of the Brillouin zone are occupied. ${ }^{8}$ Perpendicular, the self-consistently calculated wave function ${ }^{11}$ is close to $\Psi_{0}(z) \simeq \sqrt{b^{3} / 2} z e^{-b z / 2} \quad(b=3.3$ $\left.\times 10^{8} \mathrm{~m}^{-1}\right) .{ }^{12}$ Its rather extended shape leads to a $k_{z}$ distribution (Fourier transformation of $\Psi_{0}$ ) with $90 \%$ of its intensity within $0.1 \AA^{-1}$ around $\Gamma$.

(4) The surface has a very low defect density ${ }^{13}$ which reduces contributions from elastic scattering. ${ }^{14}$

(5) The $z$ extension of the 2DES of $5 \mathrm{~nm}^{8}$ is still much less than the photon wavelength $(\lambda \geqslant 40 \mathrm{~nm})$, allowing us to treat the electric field of the light as constant.

To illustrate these advantages, we use the one-step model and the knowledge that final vacuum states decay exponentially into the sample with decay length $\lambda(E) .{ }^{6}$ This allows us to derive a rather simple expression for the photoelectron intensity per photon starting with $^{7}$

$$
I(E) \propto \rho_{f}(E)\left|M_{B}(E)\right|^{2}\left|\int_{0}^{\infty} \Psi_{0}(z) e^{i k_{z}(E) z-z / \lambda(E)} d z\right|^{2} .
$$

Here, $\rho_{f}(E)$ is the relevant density of final vacuum states, $M_{B}(E)$ is the matrix element, and $k_{z}(E)$ is the wave vector of the final states inside the sample. $\Psi_{0}(z)$ is given above with $b=\left(16.5 \pi m^{\star} e^{2} N_{2 D E S} / \epsilon \epsilon_{0} \hbar^{2}\right)$, depending on effective mass $m^{\star}$, dielectric constant $\epsilon$, and the known 2DES density $N_{2 D E S}{ }^{12}$ The integral can be solved

$$
I(E) \propto \frac{\rho_{f}(E)\left|M_{B}(E)\right|^{2}}{\left\{k_{z}(E)^{2}+[b / 2+1 / \lambda(E)]^{2}\right\}^{2}},
$$

and the contributions to $I(E)$ appear rather directly. A single $k_{z}$ value is assumed, but contributions from different $k_{z}$ can be simply summed up. Since $b / 2$ and $1 / \lambda(E)$ are small with respect to the Brillouin-zone boundary at $14.6 \mathrm{~nm}^{-1}$, transitions close to $k_{z}=0$ are strongly favored. In addition, transi- 

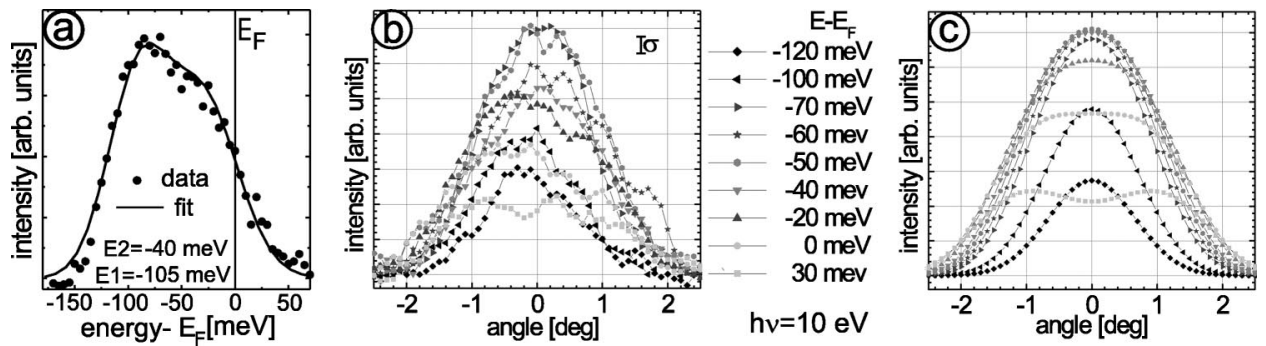

FIG. 1. (a) Energy scan of 2DES peak and fit (see text) at $h \nu=10 \mathrm{eV}, \theta=0^{\circ}, E_{\text {pass }}=4 \mathrm{eV} ; E 1$, E2: subband energies. (b) Angular scans of $2 \mathrm{DES}$ peak at different $E-E_{F}, h \nu=10 \mathrm{eV}, E_{\text {pass }}=4 \mathrm{eV}$. (C) Fit curves to (b) with same fit parameters as in (a); same symbols are used in (b) and (c) as marked in between.

tions with large $M_{B}(E)$, large $\rho_{f}(E)$, and/or large $\lambda(E)$ are most intense.

To calculate the contributions to the photocurrent quantitatively within the one-step model, one uses the time inverse of a low-energy electron diffraction (LEED) state. Its asymptotic inside vacuum is matched to the solution of the complex bulk band structure ${ }^{15}$ giving $\Phi_{L E E D}(E)$ as the final states of photoemission. To deduce $\lambda(E)$, the density of $\Phi_{L E E D}(E)$ within the crystal is fitted to $e^{-z / \lambda(E)}$. We find $\lambda(E) \in[0.9 \mathrm{~nm}, 1.7 \mathrm{~nm}]$, i.e., $1 / \lambda(E)>b / 2$. Thus, the contribution of $\lambda(E)$ is always relevant, if $k_{z}(E)$ is small.

The initial states are represented by the half-space Green's function, given as a layer resolved contribution of atomic orbitals $\Psi_{i}$. The basis set consists of $5 s$ and $5 p$ (4s and $4 p$ ) orbitals for In (As). Matrix elements $M_{B}(E)$ are calculated as $\left\langle\Phi_{L E E D}\left(E_{\mathrm{fin}}, \vec{k}_{\|}\right)\left|\vec{A}_{0} \cdot \vec{p}\right| \Psi_{i}\right\rangle$ with the light-induced vector potential $\vec{A}_{0}$ kept constant. Polarization and incident angle of the light are taken as in the experiment. Since we, thus, calculate $M_{B}(E), \lambda(E)$ and the band structure of final states, we can disentangle the different contributions to $I(E)$ given in Eq. (1).

The ARUPS experiments are performed at the HONORMI beamline of HASYLAB using the ASPHERE analyzer. ${ }^{16}$ The light is $p$ polarized with an incident angle of $30^{\circ}$ towards [001]. The energy resolution of the experiment is $20 \mathrm{meV}$ and the angular resolution is $0.25^{\circ} / 0.45^{\circ}$ in the two different directions. The Fermi level $E_{F}$ is determined on a clean Ta-foil with an accuracy of $5 \mathrm{meV}$. Degenerate $n$-InAs $\left(N_{\mathrm{D}}=1.1 \times 10^{16} \mathrm{~cm}^{-3}\right)$ is cleaved in situ at $p$ $\simeq 10^{-8} \mathrm{~Pa}$. This leads to nearly defect-free InAs(110), exhibiting flat band conditions up to the surface. ${ }^{17}$ To induce the 2DES, $4.5 \%$ of an Fe monolayer is deposited. The coverage is determined by a quartz balance and is cross-checked by a scanning tunneling microscope (STM). The surface bands shift downwards by $300 \mathrm{meV} .{ }^{8}$ One gets an accumulation layer (2DES) with $N_{2 D E S}=2 \times 10^{12} \mathrm{~cm}^{-2}$ as confirmed by scanning tunneling spectroscopy (STS). ${ }^{9}$ This gives $b=3.3$ $\times 10^{8} \mathrm{~m}^{-1}$, determining $\Psi_{0}(z)$ completely.

Figure 1(a) shows the energy distribution curve (EDC) of the 2DES at photon energy $h \nu=10 \mathrm{eV}$ and $\theta=0^{\circ}$. Figure 1(b) shows angular scans recorded at different $E-E_{F}$. A linear background caused by second-order light is subtracted. The straightforward fit of the data ${ }^{8}$ is shown as a solid line in Fig. 1(a) and in Fig. 1(c) for Fig. 1(b). The fit takes into account the known energy and angular resolution of the experiment, ${ }^{16}$ the known conduction-band dispersion of InAs (Ref. 18), and the known temperature $(300 \mathrm{~K})$. It assumes a constant $M_{B}(E), \lambda(E)$, and $\rho_{f}(E)$ as expected in the small energy and angular region of the data. The first two fitting parameters are the subband energies $E_{i}$, which are confirmed by STS. ${ }^{9}$ An adjustment of the total intensity by a single factor is still necessary, which accounts for $M_{B}(E), \lambda(E)$, and $\rho_{f}(E)$ and for the instrumental efficiency. It is the only free parameter and reproduces all results in Figs. 1(a) and 1(b).

Figure 2(a) shows the 2DES peak at different $h \nu$. The data are normalized to the photon flux calibrated by a $\mathrm{Au}$ film prior to the experiments. Therefore, the quantum efficiency of the Au diode is considered. ${ }^{19}$ During the experiments, the positron current in the synchrotron is used to link the calibrated to the actual photon flux.

Figure 2(a) shows that the normalized peak intensity depends nonmonotonously on $h \nu$. However, before we compare with calculations, we have to consider that the angular
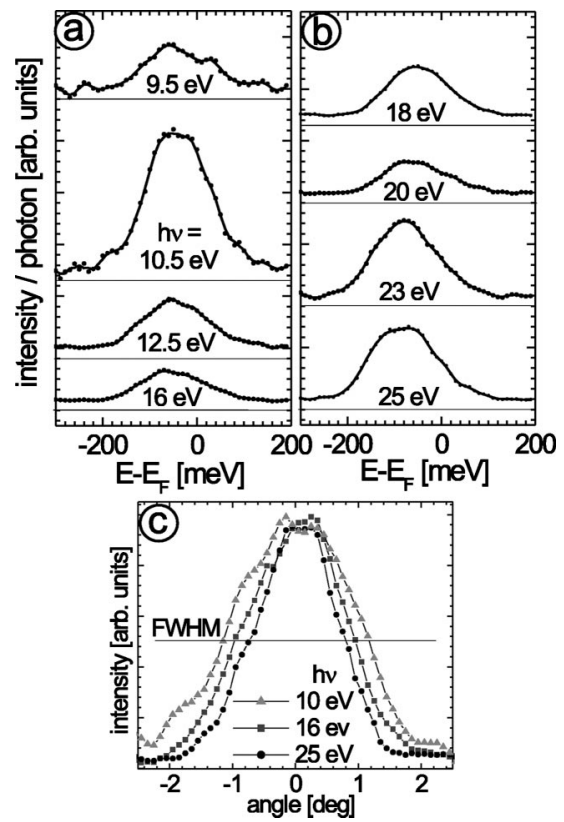

FIG. 2. (a) and (b) Energy scans of 2DES peak at different $h \nu$ as indicated, $E_{\text {pass }}=20 \mathrm{eV}, \theta=0^{\circ}$; data are scaled to photon fluence and offset as marked by horizontal lines. (c) Angular scans of 2DES peak at different $h \nu$ as indicated, $E_{\text {pass }}=4 \mathrm{eV}, E-E_{F}=-50 \mathrm{meV}$; data are scaled to the same height at $\theta=0^{\circ}$; the horizontal line [full width at half maximum (FWHM)] is at half maximum. 


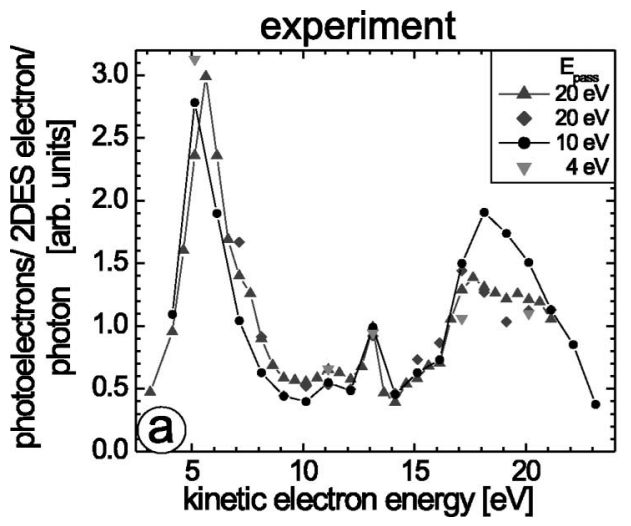

width of the peak also depends on $h \nu$. This is demonstrated in Fig. 2(c). The reason for the reduced width with increasing $h \nu$ is that $k_{\|} \propto \sin \theta \sqrt{E_{\text {kin. }}}$. Thus, the same $k$-space distribution of the 2DES leads to a smaller $\Delta \theta$ at higher photoelectron energy $E_{\text {kin }}(\propto h \nu)$, increasing the ARUPS collection efficiency. This effect can be straightforwardly calculated by the fit model described above. The normalized peak intensity devided by the collection efficiency is shown for four data sets obtained on different samples in Fig. 3(a). The statistical error is $10 \%$ at $E_{k i n}=5-19 \mathrm{eV}, 20 \%$ at higher $E_{k i n}$ and $40 \%$ at lower $E_{k i n}$. The data show a pronounced peak at $5.5 \mathrm{eV}$, a tiny peak at $13 \mathrm{eV}$ and a broad peak around $19 \mathrm{eV}$. The data are largely reproducible, except that the peak at $19 \mathrm{eV}$ is $25 \%$ larger in one data set. Note that $I(E)$ increases by a factor of 6 between $3.5 \mathrm{eV}$ and $5.5 \mathrm{eV}$.

Next, the measured $I(E)$ data will be compared with calculated $I(E)$ data. Therefore, the half-space Green's function for the initial states is calculated for $\vec{k}_{\|}=0$ and for an energy at the bottom of the InAs conduction band. Although $k_{z}$ is not a quantum number in the half-space Green's function, the restriction to the bottom of the conduction band requires $k_{z}$ $\simeq 0$ as in the experiment. A free parameter is the position of the matching plane between the vacuum LEED asymptotic and the complex states of the crystal. It is chosen at $1.4 \AA$ above the first atom, but the results do not change significantly from $1 \AA$ to $2 \AA$. Figure $3(\mathrm{~b})$ shows the calculated $I(E)$ curve. It also exhibits a sharp peak at about $6 \mathrm{eV}$ and a broad peak around $19 \mathrm{eV}$. The peak heights are nearly identical to the experiment. The tiny peak at $13 \mathrm{eV}$ faintly appears as a broad shoulder within the calculation. Generally the experimental results are nicely reproduced by the onestep model setting the base for a deeper analysis.

Figure 4 shows the different contributions to $I(E)$. The $\lambda(E)$ curve derived from $e^{-z / \lambda}$ fits to the final states is plotted in Fig. 4(a). It shows a maximum at $3.5 \mathrm{eV}$, where $\lambda$ is enhanced by $70 \%$. Setting $\lambda(E)$ into Eq. (1) with $k_{z}=0, b$ $=3.3 \times 10^{8} / \mathrm{m}, M_{B}(E)=$ const, and $\rho_{f}(E)=$ const, results in an $I(E)$ peak six times larger than the surrounding. This height is indeed found in the $I(E)$ data, but the $\lambda(E)$ peak is at $3.5 \mathrm{eV}$ and the $I(E)$ peak at $6 \mathrm{eV}$. Thus, other factors must contribute. Figure 4(b) shows $\left|M_{B}(E)\right|$, which is calculated separately for each layer. Only the As $s$-orbital is considered, since it strongly dominates the InAs conduction band. ${ }^{10} \mathrm{~A}$ strong maximum appears around $7 \mathrm{eV}$ for all layers, while a smaller maximum at $2 \mathrm{eV}$ appears only from the second
FIG. 3. (a) 2DES peak intensity normalized to the photon fluence and to the angular collection efficiency of the analyzer; different $E_{\text {pass }}$ are indicated. (b) Calculated photoelectron intensity in normal emission using the initial states at the bottom of the conduction band of InAs(110); calculation performed according to Ref. 15.

layer on. The maximum at $7 \mathrm{eV}$ is three times larger than the surrounding, which would lead to an $I(E)$ peak nine times larger than the surrounding again comparable to the $I(E)$ data. However, the $\left|M_{B}(E)\right|$ peak is at higher energy than the $I(E)$ peak. Thus, it also cannot explain the $I(E)$ peak exclusively. Finally, the $k_{z}$ distribution of the final states has to be considered. Figure 4(c) shows the complex band structure of InAs(110) in the direction perpendicular to the surface. The bulk states of an infinite crystal are displayed as thin lines, while some of the complex states decaying into the crystal are drawn as thick lines. The complex bands are marked by vertical bars, indicating their coupling strengths to vacuum. According to Eq. (1), the different $k_{z}$ contributions of the
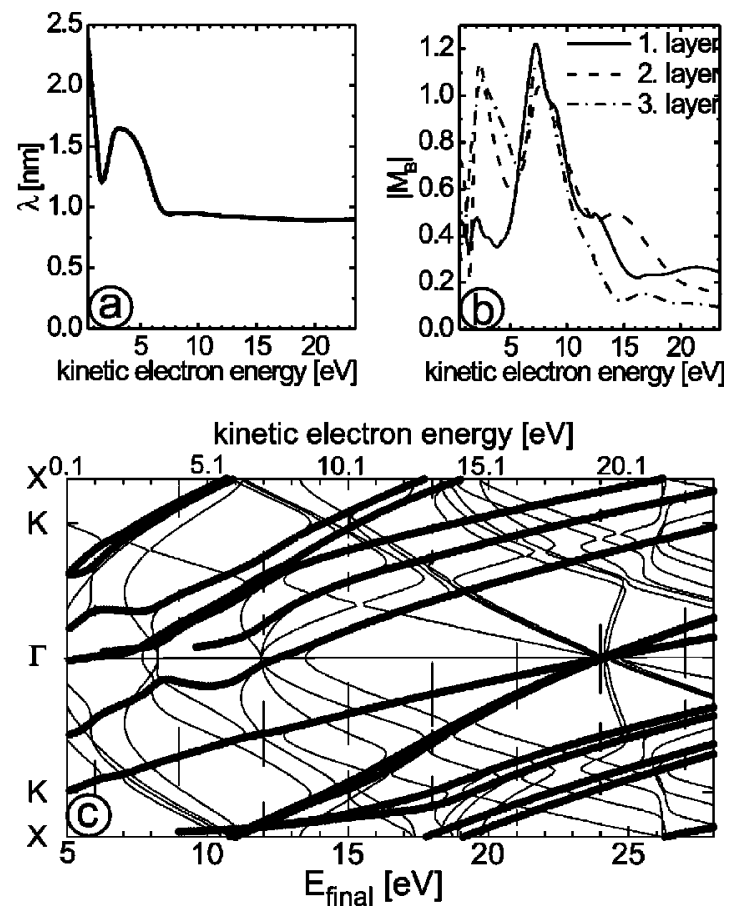

FIG. 4. (a) Calculated escape depth $\lambda$ as a function of energy. (b) Matrix elements corresponding to the As $s$ orbitals of the initial states displayed for different layers from the surface as indicated. (c) Complex band structure of InAs(110) perpendicular to the surface; thin lines are real states of the infinite crystal, thick lines are complex states of the semi-infinite crystal; vertical bars mark the strength of the coupling of complex states to vacuum states; only states with significant coupling are plotted; $E_{\text {final }}$ at the lower bar is given with respect to the valence-band maximum. 
final states have to be summed up. Obviously, the final states show neither a strong $\left(k_{z}=0\right)$ contribution at $E_{k i n}=6 \mathrm{eV}$ nor an increased density of states, i.e., the same number of bands with similar coupling strengths appear across the maximum. Thus, without performing the intricate calculation summing up over $k_{z}$ contributions, we can state that the peak at $6 \mathrm{eV}$ is due to an overlap of a peak in $M_{B}(E)$ and $\lambda(E)$, but none of the two contributions can explain it exclusively.

The second $I(E)$ peak at $19 \mathrm{eV}$ is three times larger than the surrounding $I(E)$ values. It appears neither in the $\lambda(E)$ nor in the $\left|M_{B}(E)\right|$ curves. The number of complex bands and their coupling strength to vacuum is constant around $19 \mathrm{eV}$. Moreover, the dispersion of the bands is rather linear, i.e., $\rho_{f}(E)$ is constant. However, three complex bands, including the one with the strongest coupling (largest vertical bar), cross $\Gamma$ exactly at $19 \mathrm{eV}$, giving rise to direct transitions. We conclude that this $I(E)$ peak is caused by $k_{z} \simeq 0$ of the final state. In the experiment, the overlap of the oscillating final state with $\Psi_{0}(z)$ explains the peak, while in the calculation the restriction of initial states to $k_{z} \simeq 0$ is responsible. Thus, the peaks at $6 \mathrm{eV}$ and $19 \mathrm{eV}$ have completely different origins. One is due to a large escape depth and an enhanced matrix element and the other is due to a $k_{z}$ matching of final and initial states. Note, finally, that the $I(E)$ data are only explained, if a maximum of $\lambda(E)$ is assumed. This contradicts the conventional knowledge that $\lambda(E)$ is a smooth curve with a single minimum at $50 \mathrm{eV} .^{2}$ However, it confirms pre- vious calculations on $\mathrm{GaAs}(110)$ exhibiting a similar maximum. ${ }^{6}$ The reason for the increased $\lambda$ at $3.5 \mathrm{eV}$ is not completely clear, but the bulk band structure (thin lines) exhibits a large number of extrema around $3.5 \mathrm{eV}$. Consequently, the bulk density of states is large and the complex states can couple more effectively to real states increasing their decay length. Interestingly, the high- $T_{c}$ superconductor Bi2212 also shows a $\lambda(E)$ maximum around $4 \mathrm{eV} .{ }^{20}$ However, this maximum is explained by the different plasmons of the layered material.

In summary, we measured the photoelectron intensity of a well-defined initial state given by the Fe-induced 2DES on InAs(110). The $I(E)$ curve is reproduced by a calculation within the one-step model taking the details of the experiment into account. An analysis of the calculation reveals that a peak at $E_{k i n}=5.5 \mathrm{eV}$ is due to an overlap of an increased photoelectron escape depth and an enhanced matrix element. Another peak at $E_{k i n}=19 \mathrm{eV}$ is due to a $k_{z}$ matching of initial and final states. The measurements confirm that the electron escape depth of $\operatorname{InAs}(110)$ is not a smooth function of energy but exhibits a maximum around $E_{k i n}=3.5 \mathrm{eV}$

We thank K. Rossnagel, O. Seifarth, and J. Klijn for technical assistance and acknowledge financial support from SFB 508/B4, FOR 353, and from BMBF projects 05 KS1FKB and 05 SB8 FKA7.
${ }^{1}$ A. Sekiyama, T. Iwasaki, K. Matsuda, Y. Saitoh, Y. Onuki, and S. Suga, Nature (London) 403, 396 (2000); Y. Saitoh, T. Nakatani, T. Matsushita, T. Miyahara, M. Fujisawa, K. Soda, T. Muro, S. Ueda, H. Harada, A. Sekiyama, S. Imada, H. Daimon, and S. Suga, J. Synchrotron Radiat. 5, 542 (1998).

${ }^{2}$ G. A. Somorjai, Chemistry in Two Dimensions: Surfaces (Cornell, Ithaca, 1981); G. Ertl and J. Küppers, Low Energy Electrons and Surface Chemistry (VCH, Weinheim, 1985); W. Schattke and M. A. van Hove, Solid State Photoemission and Related Methods (Wiley-VCH, Weinheim, 2003).

${ }^{3}$ D. L. Abraham and H. Hopster, Phys. Rev. Lett. 58, 1352 (1987); D. P. Pappas, K. P. Kämper, B. P. Miller, H. Hopster, D. E. Fowler, C. R. Brundle, A. C. Luntz, and Z. X. Shen, Phys. Rev. Lett. 66, 504 (1991).

${ }^{4}$ G. K. Wertheim, D. M. Riffe, N. V. Smith, and P. H. Citrin, Phys. Rev. B 46, 1955 (1992); N. V. Smith, G. K. Wertheim, A. B. Andrews, and C. T. Chen, Surf. Sci. Lett. 282, L359 (1993).

${ }^{5}$ F. R. McFeely, E. Cartier, J. A. Yarmoff, and S. A. Joyce, Phys. Rev. B 42, 5191 (1990).

${ }^{6}$ C. H. Solterbeck, O. Tiedje, T. Strasser, S. Brodersen, A. Bödicker, W. Schattke, and I. Bartos, J. Electron Spectrosc. Relat. Phenom. 101-103, 473 (1999).

${ }^{7}$ S. Hüfner, Photoelectron Spectroscopy (Springer, Berlin 1995).

${ }^{8}$ M. Morgenstern, M. Getzlaff, D. Haude, R. Wiesendanger, and R. L. Johnson, Phys. Rev. B 61, 13805 (2000).

${ }^{9}$ M. Morgenstern, J. Klijn, Chr. Meyer, M. Getzlaff, R. Adelung, R. A. Römer, K. Rossnagel, L. Kipp, M. Skibowski, and R. Wiesendanger, Phys. Rev. Lett. 89, 136806 (2002).
${ }^{10}$ J. L. Alves, J. Hebenstreit, and M. Scheffler, Phys. Rev. B 44, 6188 (1991); B. Engels, P. Richard, K. Schroeder, S. Blügel, Ph. Ebert, and K. Urban, ibid. 58, 7799 (1998); J. Klijn, L. Sacharow, Chr. Meyer, S. Blügel, M. Morgenstern, and R. Wiesendanger, ibid. 68, 205327 (2003).

${ }^{11}$ M. G. Betti, V. Corradini, G. Bertoni, P. Casarini, C. Mariani, and A. Abramo, Phys. Rev. B 63, 155315 (2001); V. Aristov, G. Le Lay, P. Soukiassian, V. M. Zhilin, G. M. Grehk, H. J. Kim, R. L. Johnson, and C. Giammichele, Surf. Rev. Lett. 5, 235 (1998).

${ }^{12}$ T. Ando, A. B. Fowler, and F. Stern, Rev. Mod. Phys. 54, 437 (1982).

${ }^{13}$ M. Morgenstern, D. Haude, V. Gudmundson, Chr. Wittneven, R. Dombrowski, Chr. Steinebach, and R. Wiesendanger, J. Electron Spectrosc. Relat. Phenom. 109, 127 (2000).

${ }^{14}$ J. Zemek, P. Jiricek, S. Hucek, A. Jablonski, and B. Lesiak, Surf. Sci. 473, 8 (2001); I. S. Tilinin, Phys. Rev. B 53, 547 (1996).

${ }^{15}$ J. Henk, W. Schattke, H. P. Barnscheidt, C. Janowitz, R. Manzke, and M. Skibowski, Phys. Rev. B 39, 13286 (1989); J. Henk, W. Schattke, H. Carstensen, R. Manzke, and M. Skibowski, ibid. 47, 2251 (1993).

${ }^{16}$ K. Rossnagel, L. Kipp, M. Skibowski, and S. Harm, Nucl. Instrum. Methods Phys. Res. A 467-8, 1485 (2001).

${ }^{17}$ C. B. M. Andersson, J. N. Andersen, P. E. S. Persson, and U. O. Karlsson, Surf. Sci. 398, 395 (1998).

${ }^{18}$ U. Merkt and S. Oelting, Phys. Rev. B 35, 2460 (1987).

${ }^{19}$ K. U. Gawlik, Ph.D. thesis, Kiel, 1996.

${ }^{20}$ M. R. Norman, M. Randeria, H. Ding, and J. C. Campuzano, Phys. Rev. B 59, 11191 (1999). 